\title{
Bystanding or standing by? \\ How the number of bystanders affects the intention to intervene in cyberbullying
}

new media \& society 2016, Vol. I8(8) 1491-1507

(C) The Author(s) 2014

Reprints and permissions:

sagepub.co.uk/journalsPermissions.nav DOI: $10.1|77 /| 46|4448| 45635$ |9 nms.sagepub.com

\author{
Magdalena Obermaier, Nayla Fawzi \\ and Thomas Koch \\ Ludwig-Maximilians-University Munich, Germany
}

\begin{abstract}
This study examines the bystander effect in cyberbullying. On the basis of two experiments, we test whether individuals who witness cyberbullying are less willing to intervene when the number of others who have already observed the incident is increased. In addition, we inquire how differently severe cyberbullying incidents affect bystanders' intention to intervene. Our results show that a very severe cyberbullying incident boosts individuals' intention to intervene, mediated by the assessment of the situation as emergency and, in turn, by an increased feeling of responsibility. However, if there is a larger number of bystanders in a cyberbullying incident, rather than just a few, participants feel less responsible to help, and thus, they are less willing to intervene.
\end{abstract}

\section{Keywords}

Bystander effect, bystander intervention, cyberbullying, diffusion of responsibility, experiments

Imagine a user observes that someone is being bullied in an online social networking site and that two others have witnessed the incident. How likely is it that this user will intervene? And now picture that more than a thousand others have already witnessed the

\section{Corresponding author:}

Magdalena Obermaier, Department of Communication Studies and Media Research, Ludwig-MaximiliansUniversity Munich, Oettingenstr. 67, 80538 Munich, Germany.

Email: obermaier@ifkw.Imu.de 
cyberbullying. Compared to the first scenario, would the user now be equally willing to help? Previous research suggests that would not be the case. As the number of bystanders observing an emergency increases, the likelihood that individuals will intervene decreases (Darley and Latané, 1968). This bystander effect is considered to be very robust in the face-to-face context. However, it still is not entirely clear whether the bystander effect also emerges in contexts of computer-mediated communication (CMC), and which variables influence the effect online (Blair et al., 2005; Palasinski, 2012).

In the present study, we analyze whether a bystander effect occurs in cyberbullying. Cyberbullying is widespread among adolescents as well as university students and highly problematic, since incidents can seriously harm the victims (Tokunaga, 2010). However, it is not only victims and perpetrators who play a decisive role in cyberbullying but also non-involved individuals who observe incidents on (semi-)public Internet applications. The behavior of these bystanders affects the perception and process of cyberbullying (Salmivalli et al., 2011). For instance, passive behavior, which occurs with most observers of such incidents, can reinforce the bullying, since a bully may perceive it as approval. In contrast, by supporting the victim, bystanders can limit harm to victims and even contribute to impeding the cyberbullying (Freis and Gurung, 2013; Kowalski et al., 2012). Since victims of cyberbullying suffer from various negative consequences, such as social exclusion and depression, and even suicidal intentions, it is extremely important to learn which variables influence bystanders' helping behavior (Bauman et al., 2013). However, while there is some research on the variables that affect the behavior of bystanders in (cyber-)bullying, the bystander effect has not yet been tested in this context (Pozzoli and Gini, 2013). Therefore, we conducted two experiments to examine whether a bystander effect occurs in cyberbullying, and which factors influence it.

\section{Cyberbullying and bystander behavior}

In this study, we define cyberbullying as use of Internet and mobile phone applications to intentionally harm, annoy, or defame others (Dooley et al., 2009; Fawzi, 2009). A single incident is included in this definition if it occurs in (semi-)public online applications, since it then has an almost unlimited reach and remains online indefinitely. Thus, victims can expose themselves several times to the bullying, and bystanders can watch or read it repeatedly, forward it to others, or even save it on their devices (Langos, 2012).

Cyberbullying can take place on almost all online applications, but as we are interested in the behavior of bystanders, we focus on (semi-)public spaces, such as Facebook groups. Most studies indicate that around $15-40 \%$ of adolescents (Smith et al., 2008; Tokunaga, 2010) and $10-40 \%$ of university students have been victims of cyberbullying (Finn, 2004; Lindsay and Krysik, 2012; MacDonald and Roberts-Pittman, 2010). Aggressive or insulting messages are one of the most common means of cyberbullying and often take place on social networking sites (Kwan and Skoric, 2013; Livingstone et al., 2013).

Bullies and victims are not the only ones involved in cyberbullying: most adolescents and young adults have already been bystanders of cyberbullying (Fawzi and Goodwin, 2011; Li, 2010; Lindsay and Krysik, 2012). Bystanders are central in shaping cyberbullying processes, as they can influence the perpetrator's behavior by either supporting the bully or the victim. Even passive behavior can have an impact, as the bully and victim 
often perceive this as a confirmation of the bullying (Kowalski et al., 2012). By getting involved, bystanders can wield effective power to stop cyberbullying and minimize the negative effects on the victim. However, studies show that most bystanders remain passive (Li, 2010; Slonje et al., 2012). Thus, to combat cyberbullying, it is important to know which factors influence bystanders' prosocial reactions. For instance, the trait of high empathy, a high incident severity, previous experiences with bullying, and the feeling of responsibility to help correlate positively with bystander intervention (Barlińska et al., 2013; Bastiaensens et al., 2014; Pozzoli and Gini, 2013; Van Cleemput et al., 2014). A common reason for not intervening appears to be a distribution of responsibility to others as bystanders frequently perceive the incidents to be none of their business (Van Cleemput et al., 2014). Accordingly, studies also show that bystanders' relationship to the bully or the victim can affect their behavior (Oh and Hazler, 2009; Macháčková et al., 2013). However, the role of the number of bystanders in cyberbullying still remains unclear.

\section{The bystander effect}

Research consistently demonstrates that the more people witness a critical situation, the less willing individuals are to intervene (Fischer et al., 2011). In the face-to-face context, this bystander effect occurs in emergencies, such as epileptic seizures, and in nonemergency situations, such as if a glass of water is accidentally spilled (Latané and Nida, 1981). The effect has been shown to be highly robust in the face-to-face context.

In order to explain what inhibits individuals from helping as the number of bystanders increases, we refer to Latané and Darley's (1970) five-step model. The model proposes the specific circumstances under which bystanders will intervene in a critical situation: first, bystanders have to notice the situation; second, they have to assess it as an emergency; third, and most importantly, they must feel responsible to intervene; fourth, they have to reflect on how to help, and in a fifth step, they need to decide to intervene and implement their decision. If any of these steps is not completed, bystanders will not get involved (Latané and Darley, 1970). Existing research has provided empirical evidence for this five-step model, although most studies rely on non-experimental data (Burn, 2009; Pozzoli and Gini, 2013).

The presence of others may hamper the completion of the proposed five-step sequence, since it particularly affects the perception of responsibility (Darley and Latané, 1968). Greater the number of bystanders, lesser the individuals feel responsible to help. This can be explained by their tendency to mentally spread the responsibility to intervene among all of the bystanders, including themselves, thus diminishing the sense of individual responsibility. In turn, this diffusion of responsibility inhibits people from intervening, establishing it as the central cause of the bystander effect. However, many studies on the bystander effect have not explicitly measured this feeling of responsibility (Darley and Latané, 1968; Fischer et al., 2011).

\section{The bystander effect in CMC}

The bystander effect states that the physical presence of others inhibits bystander intervention. However, there is preliminary evidence for a bystander effect also occurring in private and public CMC (Barron and Yechiam, 2002; Blair et al., 2005; Palasinski, 2012; 
van Bommel et al., 2012). A diffusion of responsibility is also argued to cause the effect online, but this has not been explicitly tested (Markey, 2000; Voelpel et al., 2008). Yet, as some characteristics of $\mathrm{CMC}$ differ from face-to-face interaction, feeling responsible to intervene may work differently online.

The fact that bystanders are not physically present online, along with their limited visibility, marks one key difference between face-to-face and CMC contexts. Consequently, in a CMC context, it may not be obvious how many others have already observed or are witnessing a critical situation (Blair et al., 2005). ${ }^{1}$ However, individuals could always assume that the number of others witnessing a critical incident is rather high, due to the fact that information distributed online has an almost infinite reach (Langos, 2012). Correspondingly, the reduced social cues approach states that individuals are less concerned with social norms online, since the means of direct social control for deviant behavior, such as non-verbal sanctions, are limited (Dubrovsky et al., 1991; Kiesler et al., 1984). In addition, they could be aware that it is almost impossible for other users to trace back who has observed a critical situation without intervening, which may lead users to feel more anonymous online (Polder-Verkiel, 2012; Suler, 2004). According to the theory of deindividuation, individuals then perceive less public selfawareness, which distracts them from anticipating possible negative reactions to their behavior (Postmes and Spears, 1998). These features of the online environment may occasionally boost the willingness to intervene if individuals feel responsible, since they will be less fearful of negative reactions (Hudson and Bruckman, 2004; Voelpel et al., 2008). However, being less concerned with the social consequences of their behavior, individuals may more likely feel less responsible to intervene online (Polder-Verkiel, 2012). Taken together, central CMC characteristics may, in general, lower individuals' feeling of responsibility to intervene as compared to face-to-face contexts. However, there is insufficient empirical evidence regarding whether this may affect the bystander effect in cyberbullying.

\section{Study I}

The first experiment provides an initial test of the bystander effect in cyberbullying. Hence, we expect the intention to intervene in cyberbullying to decrease as the number of bystanders observing this incident is increased (H1) (Fischer et al., 2011). In addition, we test whether a bystander effect in cyberbullying is also based upon a diffusion of responsibility. We assume that, first, the feeling of responsibility will decrease as the number of bystanders increases, and, second, that individuals rather intend not to intervene when they perceive themselves to be less responsible to help (Darley and Latané, 1968). Taken together, we predict that the number of bystanders negatively affects the intention to intervene in cyberbullying mediated by a decrease of the feeling of responsibility $(H 2)$.

\section{Method}

Participants and design. Participants were recruited using university-based E-mail distribution lists. Participation was voluntary and non-paid. A total of 85 students participated in the online experiment. Since we are interested in the influence of specific numbers of 
bystanders, we only included those in our sample who (almost) correctly recalled the number of bystanders $(n=66,80.3 \%$ female, mean age $=22.35$, standard deviation $[S D]=2.77) .^{2}$ Participants were randomly assigned to two conditions in a betweenparticipants factorial design. Groups did not differ significantly with regard to gender, $\chi^{2}(1)=1.71, p=.19$, and age, $t(64)=.34, p=.74$.

Procedure and independent variables. Participants were asked to read two (fictitious) posts on a screenshot of a university Facebook group. They should imagine being members of the group, yet not knowing any of the two members who posted comments in order to prevent possible effects from a relationship to bully or victim. The first post was written by a group member called "Michi" asking a question and requesting notes on a university lecture. The second post represented the cyberbullying incident. Therein, another group member commented on that question by harshly insulting the questioner. More concretely, the victim was called names several times, strongly insulted (e.g. "you are good for nothing and really have no business in university at all"), and all other group members were invoiced to complain about the inquirer. The stimulus appeared in the Facebook design and had all the features of Facebook groups (e.g. profile pictures of members, name of the group). However, any additional information (e.g. last names and profile pictures of bully and victim, number of members, posts' dates of dispatch) was made illegible. As independent variable, we manipulated the notification of how many people have already seen the posts. For a first test of the bystander effect in cyberbullying, we chose to compare a small number to a high number of bystanders, since there is preliminary evidence that group sizes of similar quantity (e.g. 15 or 50 bystanders) may not affect the intention to intervene differently online (Blair et al., 2005). Additionally, relying on common sizes of university Facebook groups, it was either indicated "seen by 24 " or "seen by 5025 " members.

Measures. As a treatment check, we asked participants to freely recall the number of bystanders presented above the comment in the Facebook group. In addition, they had to assess the number of bystanders on a 5-point Likert scale by rating whether "rather few" or "rather many" others have already seen the comment $(M=4.05, S D=1.00)$.

Participants also had to assess the degree of severity of the cyberbullying incident ("not threatening"_- "very threatening," "not alarming"_- "very alarming," "not dangerous"- "very dangerous," 5-point Likert scales, $\alpha=.68, M=4.29, S D=0.70$ ). The feeling of responsibility to intervene in the cyberbullying incident was measured by 3 items (5-point Likert scales): "I highly feel personally responsible to support Michi," "I consider it as my duty to help Michi," and "It is my obligation to do something about this comment" ( $\alpha=.78, M=2.44, S D=0.93)$ (Greitemeyer et al., 2006). Finally, we assessed the intention to intervene in the cyberbullying incident using the item "I would intervene" (5-point Likert scale, $M=3.04, S D=1.31$ ).

\section{Results}

Before testing the hypotheses, we conducted a treatment check. Since we preliminarily excluded participants who did not recall the number of bystanders correctly (see above), 


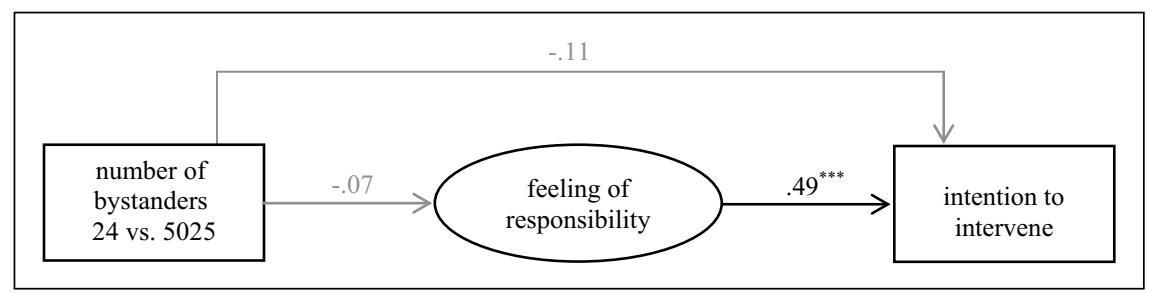

Figure I. Structural Equation Model on effects of number of bystanders on feeling of responsibility and intention to intervene.

$*_{* *} p<.001$.

Table I. Zero-order correlations (Study I).

\begin{tabular}{llll}
\hline Article I. & $\mathrm{I}$ & 2 & 3 \\
\hline I. Number of bystanders $(24$ or 5025$)$ & $\mathrm{I}$ & - & - \\
2. Feeling of responsibility & -.10 & $\mathrm{I}$ & - \\
3. Intention to intervene & -.13 & $.49 * * *$ & $\mathrm{I}$
\end{tabular}

$* * * p<.001$.

we already made sure that the experimental groups do adequately differ in terms of this recall. However, we tested whether there are group differences in regard to the relative assessment of the number of bystanders. As expected, participants considered 24 bystanders to be significantly less witnesses of the cyberbullying $(M=3.40, S D=0.91)$ than 5025 bystanders $(M=4.77, S D=0.43), t(49.30)=-7.98, p<.001, d=1.92$.

To test our assumptions, we used structural equation modeling (SEM) (Figure 1). The treatment was entered as a dummy variable into the model $(0=24$ bystanders, $1=5025$ bystanders). Zero-order correlations of all constructs included in the model are presented in Table 1. The model offered an acceptable fit for the data, $\chi^{2}(4)=6.39, p=.17$, comparative fit index $(\mathrm{CFI})=.97$, root mean square error of approximation $(\mathrm{RMSEA})=.10$, standardized root mean square residual $(\mathrm{SRMR})=.05$ (Hu and Bentler, 1999).

We assumed that people are less willing to intervene in cyberbullying the more bystanders have witnessed that incident. However, the number of bystanders did not directly affect the intention to intervene, $\beta=-.11, p=.31$. Thus, there was no support for H1. Moreover, the number of bystanders did not affect participants' feeling of responsibility, $\beta=-.07, p=.61$. For the specific numbers of bystanders used in this study, we could not demonstrate a diffusion of responsibility. Yet, we found a significant positive effect of participants' feeling of responsibility on their intention to intervene, $\beta=.49, p<.001$. Taken together, the number of bystanders had no indirect effect on the intention to intervene, $\beta_{\text {ind_B }}=-.04, p=.62$. So, there was no bystander effect mediated by the feeling of responsibility in this experiment (no support for H2). There was also no total effect of the number of bystanders on the intention to intervene, $\beta_{\mathrm{B} \_ \text {total }}=-.15, p=.23$. 


\section{Discussion}

To summarize, by comparing the effect of 24 and 5025 bystanders in cyberbullying, Study 1 revealed neither a direct nor an indirect bystander effect mediated by the feeling of responsibility. However, as predicted by Latané and Darley's (1970) model, a feeling of responsibility to get involved positively affected participants' intention to intervene. These findings raise some key questions.

First, the bystander effect may simply not emerge in cyberbullying incidents, due to some characteristics of $\mathrm{CMC}$, which could affect variables influencing the effect. It is possible, for instance, that the physical presence of bystanders is essential for the feeling of responsibility to intervene, and thus for the occurrence of a bystander effect. Due to the lack of physical presence online, along with limited visibility, bystanders may feel even more anonymous and may not fear that someone will make them responsible for not intervening. However, some studies have suggested that a bystander effect does occur online (Barron and Yechiam, 2002; Palasinski, 2012).

Second, certain numbers of bystanders may not make a difference for participants, which would explain that their intention to intervene did not continuously decline with an increasing number of bystanders (Blair et al., 2005). Although the treatment check showed that participants recognized that 5025 clearly represents a greater number of bystanders than 24 , these numbers simply may not affect their feeling of responsibility and intention to intervene, indicating a ceiling effect. This assumption is also supported by our treatment check: while participants considered 24 bystanders to be a significantly lower number of witnesses $(M=3.40, S D=0.91)$ than $5025(M=4.77, S D=0.43)$, both means are above the scale midpoint, showing that participants did not assess 24 people to be a small number of bystanders. Therefore, it seems worthwhile to test the effect again using a greater spread between the numbers of bystanders.

Third, the harmfulness of the stimulus might have affected the results. For instance, Fischer et al. (2006) showed that the bystander effect is weakened in serious emergencies. Our analysis demonstrates that participants considered the incident presented to be rather severe ( 24 bystanders: $M=4.17, S D=0.74 ; 5025$ bystanders: $M=4.41, S D=.65$ ), $t(64)=-1.44, p=.16, d=0.35$. The severity of the cyberbullying incident might increase the assessment of the situation as an emergency which, in turn, could affect participants' feeling of responsibility to intervene (Latané and Darley, 1970). Therefore, a follow-up study should vary both the number of bystanders and the degree of severity of the cyberbullying incident more markedly.

\section{Study 2}

The second study sought to extend the findings of Study 1 both by using more diverse numbers of bystanders and varying the degree of severity of cyberbullying. Therefore, we again suppose that the intention to intervene in an incident of cyberbullying decreases as the number of bystanders is increased (H1). Furthermore, we again predict that the number of bystanders negatively affects the intention to intervene in cyberbullying mediated through a decrease of the feeling of responsibility (H2) (Darley and Latané, 1968). 


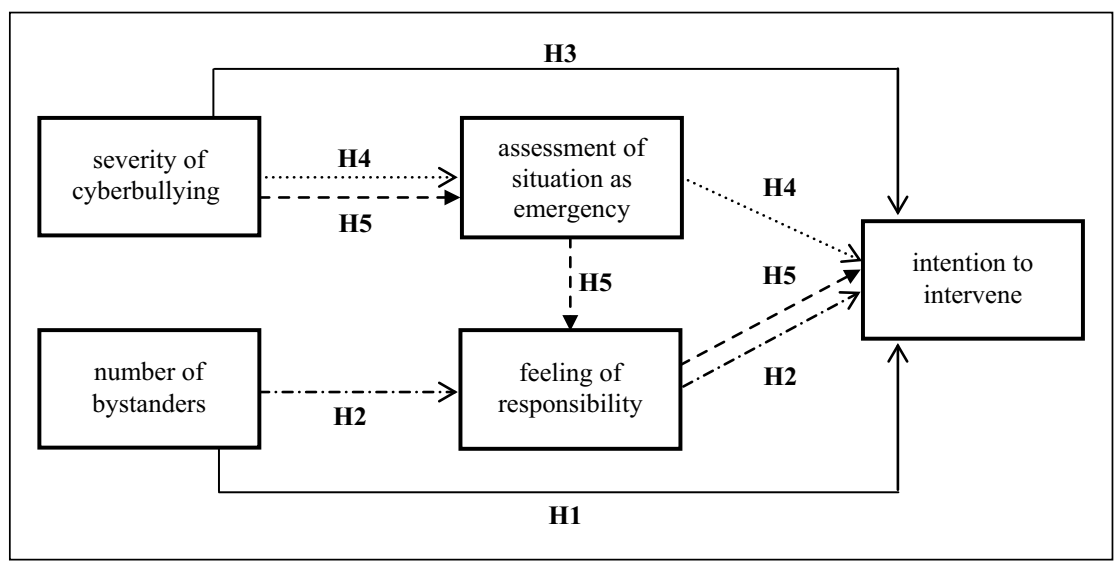

Figure 2. Postulated model on effects of number of bystanders and severity of cyberbullying on intention to intervene.

Research shows that bystanders are more willing to help in severe emergencies in the face-to-face context. This may be due to the fact that in severe emergencies, it is more evident that the victim needs help (Clark and Word, 1972). Therefore, we suppose that the more severe a cyberbullying incident is, the more individuals intend to intervene (H3). We also assume that a more severe cyberbullying incident will be assessed as a more severe emergency and that individuals are more willing to intervene accordingly (Clark and Word, 1972). Taken together, we predict that the severity of a cyberbullying incident will positively affect the intention to intervene mediated through the assessment of this situation as an emergency (H4). Based on Latané and Darley's (1970) model, we presume that the more severe individuals assess an emergency, the more they feel responsible to intervene. Hence, we predict another indirect effect: the severity of a cyberbullying incident positively affects the intention to intervene mediated through the assessment of this situation as an emergency and, in turn, by an increase of individuals' feeling of responsibility (H5) (Figure 2).

Thus, we tested three of the five steps proposed in the model of Latané and Darley (1970). The first step was not considered here, since we were foremost interested in experimentally testing the bystander effect. By presenting cover and stimulus, we automatically assured that participants noticed the critical situation. The fourth step, deciding in which form to help best, was also excluded. Since we provide a first test of the bystander effect in cyberbullying, we were interested in the overall intention to intervene regardless the exact way of helping.

\section{Method}

Participants and design. We recruited a total of 440 students by using an Online Access Panel for social science research. Participation was voluntary and non-paid. Again, the sample only included participants who (almost) correctly recalled the number of 
bystanders presented ( $n=266,68.9 \%$ female, age: $M=23.98, S D=3.20) .{ }^{3}$ Participants were randomly assigned to eight conditions in a 4 (number of bystanders: 2, 24, 224, or $5025) \times 2$ (severity of the cyberbullying: medium or high) between-participants design. Experimental conditions did not differ significantly in terms of gender, $\chi^{2}(7)=6.57$, $p=.48$, and age, $F(7,251)=.79, p=.60$.

Procedure and independent variables. The procedure of this study was similar to that of the previous experiment. Again, participants were exposed to a cyberbullying scenario in a university Facebook group, where one group member posted a question and another member commented on it in a rude way.

Since we decided to use more diverse numbers of bystanders, we relied on Voelpel et al.'s (2008) categorization, who preliminary tested the bystander effect in online communities of small, medium, large, and very large size. In addition, for 24 bystanders were not perceived as a small number of bystanders for a Facebook group in Study 1, we now used two bystanders as a small number. Therefore, participants were assigned to one of four different bystander-conditions: Above the posts was either indicated that $2,24,224$, or 5025 other members have already seen the incident. As a second factor, we varied the degree of severity of the cyberbullying incident by manipulating the degree of threat and harm for the victim (Shotland and Huston, 1979). We constructed a medium severe and a highly severe version: the comment contained defamations and insults in both versions. The highly severe version was the same as the stimulus presented in Study 1. The victim was called names several times, insulted very harshly, and all readers were invoiced to complain about the inquirer. The medium severe version was quite similar, but contained more subtle and rather mild insults (e.g. 'writing you were 'ill' and could not make it to the lecture is a really original excuse, well done!").

Measures. Participants again had to recall the number of bystanders presented and to assess whether a relatively small or a relatively high number of bystanders has already seen the two posts (5-point Likert scale, $M=3.59, S D=1.38$ ). Participants should also rate the degree of severity of the cyberbullying (5-point Likert scales, "not threatening""very threatening," "not alarming"_- "very alarming," "not dangerous"_- "very dangerous," $\alpha=.83, M=3.64, S D=1.04)$.

In addition, we measured the assessment of the situation as emergency by inquiring participants' agreement to the items "this comment constitutes a threat for inquirer Michi," "the commentator's statements harm Michi," and "the comment results in negative consequences for Michi" (5-point Likert scales, 1= "absolutely not applicable" to $5=$ "absolutely applicable," $\alpha=.81, M=3.22, S D=1.02$ ) (Shotland and Huston, 1979).

Again, participants indicated their feeling of responsibility by the 3 items used in the first experiment (5-point Likert scales, "I highly feel personal responsible to support Michi," "I consider it as my duty to help inquirer Michi," "It is my obligation to do something about this comment," $\alpha=.87, M=2.23, S D=1.03$ ) (Greitemeyer et al., 2006). Finally, participants addressed their intention to intervene in the cyberbullying incident (5-point Likert scale, "I would intervene," $M=2.77, S D=1.28$ ). 


\section{Results}

Treatment check. To check whether our treatment worked adequately, we first tested the relative assessment of the number of bystanders in the four conditions. The treatment check revealed that as the number of bystanders ascended, participants correspondingly stated that an increasing number of others has witnessed the incident of cyberbullying, $F(3,262)=137.25, p<.001, \eta^{2}=.61$. As expected, two bystanders were indeed perceived as very few $(M=1.79, S D=1.03$ on a 5-point Likert scale), 24 bystanders already as rather many $(M=3.53, S D=1.03), 224$ bystanders as quite a lot $(M=4.30, S D=0.77)$, and 5025 as a very large number of bystanders $(M=4.67, S D=0.51$, all means differ significantly, $p<.05$, Games-Howell). Hence, the treatment was successful.

For the second part of our treatment check, we compared participants' perception of the severity of the cyberbullying incident. As expected, participants rated the medium severe incident of cyberbullying as significantly less threatening, alarming, and dangerous $(M=3.10, S D=0.94)$ than the highly severe incident $(M=4.13, S D=0.87)$, $t(264)=-9.21, p<.001, d=1.13$. Thus, this treatment also worked reasonably well.

Effects of the number of bystanders and the severity of cyberbullying on the assessment of the emergency and the feeling of responsibility. Before testing the postulated model, we analyzed the direct effects of the treatment. This is an essential preliminary step to check how the constructs are related to each other (e.g. linear). We computed an analysis of variance (ANOVA) on the agreement that the incident constitutes an emergency with the degree of severity and the number of bystanders as independent variables. The analysis revealed a main effect of the incident's degree of severity, $F(1,258)=24.46, p<.001$, $\eta^{2}=.09$. As expected, participants perceived the more severe cyberbullying as a significantly more severe emergency for the victim $(M=3.51, S D=1.02)$ than the incident constructed as medium severe $(M=2.91, S D=0.93)$. In contrast, neither a significant main effect emerged from the number of bystanders, $F(3,258)=0.24, p=.87, \eta^{2}=.003$, nor a significant interaction, $F(3,258)=.05, p=.98, \eta^{2}=.001$.

We computed a second ANOVA on participants' feeling of responsibility with the degree of severity and the number of bystanders as independent variables. The ANOVA yielded a weak, yet significant main effect of the number of bystanders, $F(3$, $258)=4.52, p=.004, \eta^{2}=.05$. The pairwise comparisons showed that if only two bystanders have witnessed the cyberbullying, participants considered themselves to be more responsible to intervene $\left(M=2.50^{\mathrm{a}}, S D=1.08\right)$ than with $24\left(M=1.95^{\mathrm{b}}\right.$, $S D=0.92)$, or 5025 others watching $\left(M=2.12^{\text {b,c }}, S D=0.99\right.$, significant group differences are labelled with different identification letters). Yet, the feeling of responsibility did not significantly differ when two or 224 bystanders have seen the cyberbullying incident $\left(M=2.37^{\mathrm{a}, \mathrm{c}}, S D=1.06\right)$. To sum it up, the feeling of responsibility did not linearly decrease when the number of bystanders increased. The analysis further revealed a weak main effect of the cyberbullying's degree of severity on the feeling of responsibility, $F(1,258)=9.03, p=.003, \eta^{2}=.03$. No significant interaction between the number of bystanders and the degree of severity emerged, $F(3,258)=0.34, p=.80$, $\eta^{2}=.004$. 
Table 2. Zero-order correlations (Study 2).

\begin{tabular}{llllll}
\hline & 1 & 2 & 3 & 4 & 5 \\
\hline I. Severity of cyberbullying & 1 & & & \\
2. Number of bystanders (2 or 5025) & .09 & 1 & & \\
3. Assessment of situation as emergency & $.32^{* * *}$ & .08 & 1 & & \\
4. Feeling of responsibility & $.16^{* *}$ & $-.18^{*}$ & $.45^{* * *}$ & 1 \\
5. Intention to intervene & $.20^{* * *}$ & -.14 & $.26^{* * *}$ & $.52^{* * *}$ & I \\
\hline
\end{tabular}

$*_{p}<.05 ; * * p<.01 ; * * * p<.001$.

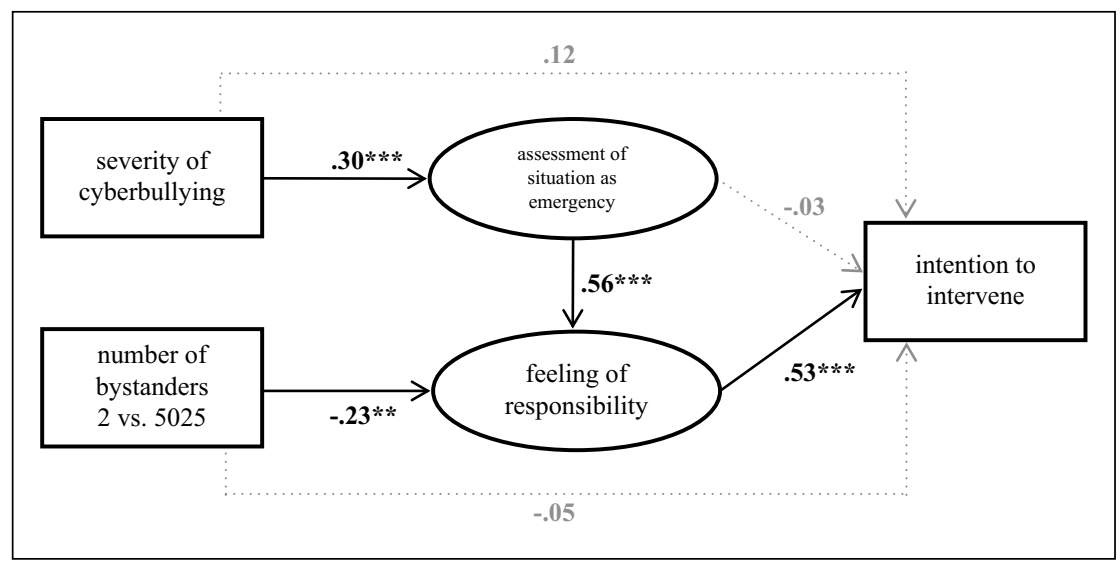

Figure 3. Structural Equation Model on effects of number of bystanders and severity of cyberbullying on intention to intervene.

$*^{* *} p<.01$; *** $p<.001$.

Effects of the number of bystanders and the severity of cyberbullying on the intention to intervene. We used SEM to test the proposed model. Since the ANOVA showed that the feeling of responsibility was lower when only two bystanders have been present compared to higher numbers of bystanders, but also demonstrated that there was no linear relationship between the number of bystanders and the feeling of responsibility, we dummy coded this independent variable. Although the largest mean differences were between 2 and 24 bystanders, we decided to compare the two groups that differed most with regard to the treatment check. Thus, we included the group with two bystanders (coded as 0 ) and the group with 5025 bystanders (coded as 1) in our analysis. We also included the incident's degree of severity in our SEM $(0=$ medium severe, $1=$ highly severe). Zero-order correlations of all constructs represented in the model are presented in Table 2. The model offered a good fit for the data, $\chi^{2}(22)=31.60, p=.08$; CFI $=.98$; $\mathrm{RMSEA}=.06, \mathrm{SRMR}=.04$ (Hu and Bentler, 1999).

Figure 3 shows the structural equation results. As in Study 1, no direct effect of the number of bystanders on the intention to intervene emerged, $\beta=-.05, p=.55$. Thus, $H 1$ 
was again rejected. However, participants considered themselves more responsible to intervene as two bystanders instead of 5025 have already observed the cyberbullying, $\beta=-.23, p=.004$. In turn, feeling responsible boosted participants' intention to intervene, $\beta=.53, p<.001$. In a next step, we tested the mediation hypothesis: There was indeed a significant negative indirect effect of the number of bystanders on participants' intention to intervene mediated by the feeling of responsibility, $\beta_{\text {ind_B }}=-.12, p=.01$. Hence, $H 2$ was confirmed in this experiment. Taken together, when comparing 2 and 5025 bystanders of cyberbullying, the experiment showed a total bystander effect of $\beta_{\text {total_B }}=-.17, p=.04$.

The severity of the cyberbullying incident did not directly affect the intention to intervene, $\beta=.12, p=.13$ (no support for $H 3$ ). Yet, it raised the assessment of the situation as an emergency, $\beta=.30, p<.001$. However, this assessment did not affect the intention to intervene, $\beta=-.03, p=.79$. Thus, there was no significant indirect effect of the severity of cyberbullying on participants' intention to intervene mediated through the assessment of this situation as an emergency, $\beta_{\text {ind } \_s_{-1}}=-.01, p=.79$. Hence, $H 4$ was rejected. However, participants felt more responsible, the more severe they rated the emergency for the victim, $\beta=.56, p<.001$. Thus, the severity of cyberbullying affected the intention to intervene by strengthening the perception that the incident marks an emergency, what boosted participants' feeling of responsibility in turn, $\beta_{\text {ind } \_s \_2}=.09, p=.01$, confirming $H 5$. In total, there was a positive indirect effect of the severity of cyberbullying on participants' intention to intervene, $\beta_{\text {ind total } \mathrm{S}}=.08, p=.03$. Summed up, we identified two counteracting effects: The severity of cyberbullying positively affected the intention to intervene, $\beta_{\text {total } \_s}=.20 p=.01$, whereas the presence of others inhibited this intention, $\beta_{\text {total_B }}=-.17, p=.04$.

\section{Discussion}

Although there is no support for a direct bystander effect, Study 2 revealed an indirect bystander effect by comparing a very small and a very large number of bystanders, mediated by the feeling of responsibility. Therefore, the first key finding of Study 2 was that when a very large number of bystanders have been present, the feeling of responsibility remained at a low level and simultaneously weakened participants' intention to react. Study 2 also provided experimental evidence that participants will assess the cyberbullying to be an emergency when a highly severe incident is presented. That, in turn, made them feel more responsible and raised their intention to intervene, as proposed in Latané and Darley's (1970) model. Thus, the second key result of Study 2 is that two opposing effects alongside the proposed model could be identified: Whereas a highly severe incident of cyberbullying positively affected participants' intentions to intervene, these intentions were inhibited by the presence of a large number of bystanders.

\section{General discussion}

\section{Discussion of the results}

As the number of bystanders in a critical situation rises, individuals are less willing to help. So far, there has only been singular evidence that this bystander effect emerges 
online (Blair et al., 2005; Palasinski, 2012). In cyberbullying, the bystander effect has not been tested at all — despite the fact that, given the importance of understanding which variables influence bystanders' behavior, this is an extremely relevant concern. Thus, this study provides an important step forward in the understanding of bystander behavior in cyberbullying, as well as the emergence of a bystander effect online.

The current work showed no classic bystander effect in cyberbullying, meaning that neither participants' feeling of responsibility nor their intention to intervene linearly decreased with an increasing number of bystanders (Fischer et al., 2011; Latané and Darley, 1970). Accordingly, comparing 24 versus 5025 bystanders in Study 1, we found that the number of bystanders neither affected the bystanders' intention to intervene nor their feeling of responsibility. Expanding these results in Study 2 by increasing the spread in the number of bystanders, we showed that there was no linear relationship between the number of bystanders and the feeling of responsibility. However, the feeling of responsibility decreased when a very small number ( 2 bystanders) was compared to a medium ( 24 bystanders) or a large number of bystanders ( 5025 bystanders), hence weakening participants' intention to intervene. Given the fact that a direct effect on the intention to intervene did not emerge, this indirect bystander effect was fully mediated by the feeling of responsibility. Therefore, we explicitly demonstrated that the diffusion of responsibility is indeed a key variable for the occurrence of this specific bystander effect in cyberbullying. Additionally, even if participants rated the relative numbers of bystanders to be diverse, the presence of a high number of bystanders did not necessarily make a difference in their feeling of responsibility to intervene. The fact that only certain group sizes have an impact on bystanders' intention to intervene may be characteristic of cyberbullying in (semi-)public online environments (Blair et al., 2005). Therefore, further research is needed to thoroughly understand the influence of certain numbers of bystanders on prosocial behavioral intentions online.

As previous research has revealed that the severity of an emergency affects bystanders' intentions to intervene, we also varied the severity of the cyberbullying in Study 2 . Thereby, we experimentally traced decisive steps of the model of Latané and Darley (1970) in cyberbullying for the first time. Importantly, we found two opposing effects: a highly severe cyberbullying incident positively affected participants' feeling of responsibility and, in turn, their intentions to intervene. However, the presence of a large number of bystanders as included in the model lowered bystanders' feelings of responsibility to act, and therefore hampered their intention to intervene.

\section{Limitations}

Certain limitations of the study must be addressed. First, we confronted participants with cyberbullying within a highly controlled setting: participants had to imagine that they were part of a university Facebook group where a cyberbullying incident took place, but that they did not know any of the group members personally. This constituted a rather artificial situation. Furthermore, to explicitly clarify the influence of specific numbers of bystanders, we used the indication of the number of bystanders as provided in Facebook groups. We could not fully rule out that participants did not assume that the cyberbullying had already been shared. In addition, since we for the first time tested effects of diverse 
numbers of bystanders in cyberbullying, we only included participants in our sample who at least approximately recalled the number of bystanders correctly. Taking these considerations into account, the influence of the number of bystanders may have been overestimated.

Second, we used a specific stimulus material. We chose insults as incident and used a Facebook group as its setting, since research demonstrates that cyberbullying often marks harsh insults on social networking sites (Livingstone et al., 2013). However, we could not be completely sure to what extent the results outlined above were influenced by this specific (semi-)public context. In addition, since the cyberbullying was in response to a question by the victim, bystanders might also partly blame the victim for asking and thus be overall less willing to intervene (Weber et al., 2013). However, the question was constructed as harmless and highly legitimate, in order to decrease the likelihood of those opinions arising.

Third, we used rather small and homogenous samples of students; this also limits our results in various respects. Thus, future studies should try to replicate and extend our findings by using larger and differently composed samples (e.g. children, adolescents).

Fourth, experimentally testing the bystander effect and decisive steps of Latané and Darley's (1970) model preliminarily in cyberbullying, we focused on situational variables included in the model. Therefore, we did not consider personal variables, such as participants' empathy for the victim (Van Cleemput et al., 2014). Moreover, measuring the intention to intervene instead of actual helping behavior marks a strong restriction to the results. Hence, although we assume that the intention to intervene positively correlates with intervention behavior, actual behavior should be tested as a dependent measure in future studies. However, confronting participants with cyberbullying and demanding a decision as whether or not to actually intervene may be critical from an ethical perspective (Freis and Gurung, 2013). Furthermore, using SEM, causality can only be confirmed regarding the influence of the two experimental factors on the directly linked dependent measures. However, the remaining paths have been theoretically outlined and also been demonstrated in several contexts (Anker and Feeley, 2011; Burn, 2009).

\section{Future research directions}

There are a number of remaining questions and avenues for further research. First, using different forms of cyberbullying might provide deeper insights into the influence of the number of bystanders. Second, the evidence that small versus larger numbers of bystanders make a difference for the feeling of responsibility to intervene should be tested again in order to assure that this is not an outlier event. If this finding is confirmed, researchers may need to rethink some theoretical considerations concerning the bystander effect online. Third, Latané and Darley's (1970) model, including the number of bystanders, could be retested with adolescents as cyberbullying usually peaks during this age. For instance, due to different stages of personality development, developmental tasks, and different roles of peers during adolescence, our results cannot be transferred to this age group. Fourth, future research should include other situational (e.g. victim asking for help) as well as personal (e.g. bystanders' relationship to bully or victim) factors, which may positively influence the feeling of responsibility and thereby prevent a bystander effect. 


\section{Funding}

This research received no specific grant from any funding agency in the public, commercial, or not-for-profit sectors.

\section{Notes}

1. However, there are applications that mark how many others have already seen or shared a post (e.g. Facebook) or clicked on a video (e.g. YouTube).

2. Participants either had to recall the exact number of bystanders presented or their indication had to be about $20 \%$ below or above the correct number of bystanders.

3. The criteria for an (almost) correct recall were the same as in Study 1.

\section{References}

Anker AE and Feeley TH (2011) Are nonparticipants in prosocial behavior merely innocent bystanders? Health Communication 26: 13-24. DOI:10.1080/10410236.2011.527618.

Barlińska J, Szuster A and Winiewski M (2013) Cyberbullying among adolescent bystanders: role of the communication medium, form of violence, and empathy. Journal of Community \& Applied Social Psychology 23: 37-51. DOI:10.1002/casp.2137.

Barron G and Yechiam E (2002) Private e-mail requests and the diffusion of responsibility. Computers in Human Behavior 18: 507-520. DOI:10.1016/S0747-5632(02)00007-9.

Bastiaensens S, Vandebosch H, Poels K, et al. (2014) Cyberbullying on social network sites. An experimental study into bystanders' behavioural intentions to help the victim or reinforce the bully. Computers in Human Behavior 31: 259-271. DOI:10.1016/j.chb.2013.10.036.

Bauman S, Toomey RB and Walker JL (2013) Associations among bullying, cyberbullying, and suicide in high school students. Journal of Adolescence 36: 341-350. DOI:10.1016/j. adolescence.2012.12.001.

Blair CA, Thompson LF and Wuensch KL (2005) Electronic helping behavior: the virtual presence of others makes a difference. Basic and Applied Social Psychology 27: 171-178. DOI:10.1207/s15324834basp2702 8.

Burn SM (2009) A situational model of sexual assault prevention through bystander intervention. Sex Roles 60: 779-792. DOI:10.1007/s11199-008-9581-5.

Clark RD and Word LE (1972) Why don't bystanders help? Because of ambiguity? Journal of Personality and Social Psychology 24: 392-400. DOI:10.1037/h0033717.

Darley JM and Latané B (1968) Bystander intervention in emergencies: diffusion of responsibility. Journal of Personality and Social Psychology 8: 377-383. DOI:10.1037/h0025589.

Dooley JJ, Pyzalski J and Cross D (2009) Cyberbullying versus face-to-face bullying. Zeitschrift für Psychologie/Journal of Psychology 217: 182-188. DOI:10.1027/0044-3409.217.4.182.

Dubrovsky VJ, Kiesler S and Sethna BN (1991) The equalization phenomenon: status effects in computer-mediated and face-to-face decision-making groups. Human-Computer Interaction 6: 119-146. DOI:10.1207/s15327051hci0602 2.

Fawzi N (2009) Cyber-Mobbing. Ursachen und Auswirkungen von Mobbing im Internet [Cyberbullying. Causes and Consequences of Bullying on the Internet]. Baden-Baden: Nomos.

Fawzi N and Goodwin B (2011) Witnesses of the offense. What influences the behavior of bystanders of cyberbullying? Paper presented at the 61st annual conference of the International Communication Association (ICA), Boston, MA, 26-30 May.

Finn J (2004) A survey of online harassment at a university campus. Journal of Interpersonal Violence 19: 468-483. DOI:10.1177/0886260503262083. 
Fischer P, Greitemeyer T, Kastenmüller A, et al. (2011) The bystander-effect: a meta-analytic review on bystander intervention in dangerous and non-dangerous emergencies. Psychological Bulletin 137: 517-537. DOI:10.1037/a0023304.

Fischer P, Greitemeyer T, Pollozek F, et al. (2006) The unresponsive bystander: are bystanders more responsive in dangerous emergencies? European Journal of Social Psychology 36: 267-278. DOI:10.1002/ejsp.297.

Freis SD and Gurung RAR (2013) A Facebook analysis of helping behavior in online bullying. Psychology of Popular Media Culture 2: 11-19. DOI:10.1037/a0030239.

Greitemeyer T, Fischer P, Kastenmüller A, et al. (2006) Civil courage and helping behavior: differences and similarities. European Psychologist 11: 90-98. DOI:10.1027/1016-9040.11.2.90.

$\mathrm{Hu}$ L-T and Bentler PM (1999) Cutoff criteria for fit indexes in covariance structure analysis: conventional criteria versus new alternative. Structural Equation Modeling 6: 1-55. DOI:10.1080/10705519909540118.

Hudson JM and Bruckman AS (2004) The bystander effect: a lens for understanding patterns of participation. The Journal of the Learning Sciences 13: 165-195. DOI:10.1207/s153278-09j1s1302_2.

Kiesler S, Siegel J and McGuire TW (1984) Social psychological aspects of computermediated communication. American Psychologist 39: 1123-1134. DOI:10.1037//0003066X.39.10.1123.

Kowalski RM, Limber SP and Agatston PW (2012) Cyberbullying. Bullying in the Digital Age. Malden, MA: Wiley-Blackwell.

Kwan GCE and Skoric MM (2013) Facebook bullying: an extension of battles in school. Computers in Human Behavior 29: 16-25. DOI:10.1016/j.chb.2012.07.014.

Langos C (2012) Cyberbullying: the challenge to define. CyberPsychology, Behavior, and Social Networking 15: 285-289. DOI:10.1089/cyber.2011.0588.

Latané B and Darley JM (1970) The Unresponsive Bystander: Why Doesn't He Help? New York: Appleton-Century-Crofts.

Latané B and Nida S (1981) Ten years of research on group size and helping. Psychological Bulletin 89: 308-324. DOI:10.1037//0033-2909.89.2.308.

Li Q (2010) Cyberbullying in high schools: a study of students' behaviors and beliefs about this new phenomenon. Journal of Aggression, Maltreatment \& Trauma 19: 372-392. DOI:10.1080/10926771003788979.

Lindsay M and Krysik J (2012) Online harassment among college students. A replication incorporating new Internet trends. Information, Communication \& Society 15: 703-719. DOI:10.10 80/1369118X.2012.674959.

Livingstone S, Ólafsson K and Staksrud E (2013) Risky social networking practices among "underage" users: lessons for evidence-based policy. Journal of Computer-Mediated Communication 18: 303-320. DOI:10.1111/jcc4.12012.

MacDonald CD and Roberts-Pittman B (2010) Cyberbullying among college students: prevalence and demographic differences. Procedia: Social and Behavioral Sciences 9: 2003-2009. DOI:10.1016/j.sbspro.2010.12.436.

Macháčková H, Dedkova L, Sevcikova A, et al. (2013) Bystanders' support of cyberbullied schoolmates. Journal of Community \& Applied Social Psychology 23: 25-36. DOI:10.1002/casp.2135.

Markey PM (2000) Bystander intervention in computer-mediated communication. Computers in Human Behavior 16: 183-188. DOI:10.1016/S0747-5632(99)00056-4.

Oh I and Hazler RJ (2009) Contributions of personal and situational factors to bystanders' reactions to school bullying. School Psychology International 30: 291-310. DOI:10.1177/ 0143034309106499.

Palasinski M (2012) The roles of monitoring and cyberbystanders in reducing sexual abuse. Computers in Human Behavior 28: 2014-2022. DOI:10.1016/j.chb.2012.05.020. 
Polder-Verkiel SE (2012) Online responsibility: bad samaritanism and the influence of internet mediation. Science and Engineering Ethics 18: 117-141. DOI:10.1007/s11948-010-9253-z.

Postmes T and Spears R (1998) Deindividuation and antinormative behavior: a meta-analysis. Psychological Bulletin 123: 238-259. DOI:10.1037//0033-2909.123.3.238.

Pozzoli T and Gini G (2013) Why do bystanders of bullying help or not? A multidimensional model. Journal of Early Adolescence 33: 315-340. DOI:10.1177/0272431612440172.

Salmivalli C, Voeten M and Poskiparta E (2011) Bystanders matter: associations between reinforcing, defending, and the frequency of bullying behavior in classrooms. Journal of Clinical Child \& Adolescent Psychology 40: 668-676. DOI:10.1080/15374416.2011.597090.

Shotland RL and Huston TL (1979) Emergencies: what are they and do they influence bystanders to intervene? Journal of Personality and Social Psychology 37: 1822-1834. DOI:10.1037//00223514.37.10.1822.

Slonje R, Smith PK and Frisén A (2012) Processes of cyberbullying, and feelings of remorse by bullies: a pilot study. European Journal of Developmental Psychology 9: 244-259. DOI:10. 1080/17405629.2011.643670.

Smith PK, Mahdavi J, Carvalho M, et al. (2008) Cyberbullying: its nature and impact in secondary school pupils. Journal of Child Psychology and Psychiatry 49: 376-385. DOI:10.1111/ j.1469-7610.2007.01846.x.

Suler J (2004) The online disinhibition effect. CyberPsychology \& Behavior 7: 321-326. DOI:10.1089/1094931041291295.

Tokunaga RS (2010) Following you home from school: a critical review and synthesis of research on cyberbullying victimization. Computers in Human Behavior 26: 277-287. DOI:10.1016/j. chb.2009.11.014.

Van Bommel M, van Prooijen J-W, Elffers H, et al. (2012) Be aware to care: public self-awareness leads to a reversal of the bystander effect. Journal of Experimental Social Psychology 48: 926-930. DOI:10.1016/j.jesp.2012.02.011.

Van Cleemput K, Vandebosch H and Pabian S (2014) Personal characteristics and contextual factors that determine "helping," "joining in," and "doing nothing" when witnessing cyberbullying. Aggressive Behavior 40: 383-396. DOI:10.1002/ab.21534.

Voelpel SC, Eckhoff RA and Förster J (2008) David against Goliath? Group size and bystander effects in virtual knowledge sharing. Human Relations 61: 271-295. DOI:10.1177/ 0018726707087787.

Weber M, Ziegele M and Schnauber A (2013) Blaming the victim: the effects of extraversion and information disclosure on guilt attributions in cyberbullying. Cyberpsychology, Behavior, and Social Networking 16: 254-259. DOI:10.1089/cyber.2012.0328.

\section{Author biographies}

Magdalena Obermaier is a research associate at the Department of Communication Studies and Media Research, University of Munich, Germany. Her research focuses on media effects, effects of persuasive communication, and the relationship between journalism and public relations.

Nayla Fawzi is a research associate at the Department of Communication Studies and Media Research, University of Munich, Germany. Her research focuses on the mediatization of politics, political socialization, and cyberbullying.

Thomas Koch is a research associate at the Department of Communication Studies and Media Research, University of Munich, Germany. His research focuses on effects of persuasive communication, the relationship between journalism and public relations, media use, and political communication. 